\title{
Feuer und Flamme für Brandschutzvorschriften
}

\section{Strafrechtliche Sorgfaltspflichten im Umgang mit Feuergefahren}

\author{
Marc Thommen / Martina Farag-Jaussi *
}

Selbstentzündliche Putzmittel, eingeschaltete Herdplatten, Holzofenheizungen mit Funkenaustritt - Brandgefahren sind ein ständiger Begleiter unseres Alltags. Kommt es in Privathaushalten zu einem ungewollten Brand mit Sach-oder Personenschaden, stellt sich die Frage, ob der Tatbestand der fahrlässigen Verursachung einer Feuersbrunst erfüllt ist. Der vorliegende Beitrag gibt einen Überblick über die Voraussetzungen von Art. 222 StGB und beleuchtet kritisch die Herkunft von und Mindestanforderungen an besondere Sorgfaltsnormen.

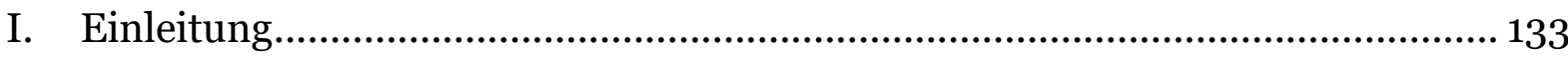

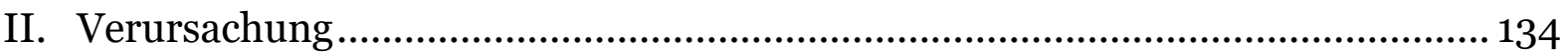

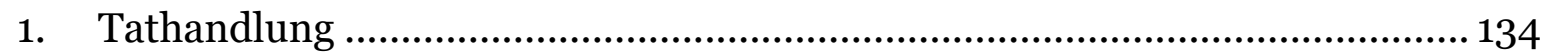

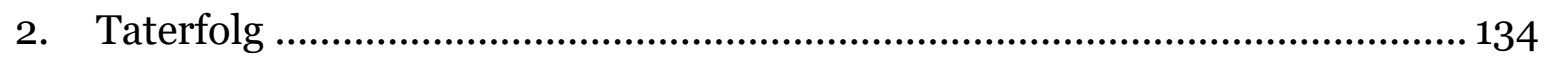

3. Kausalität................................................................................................. 135

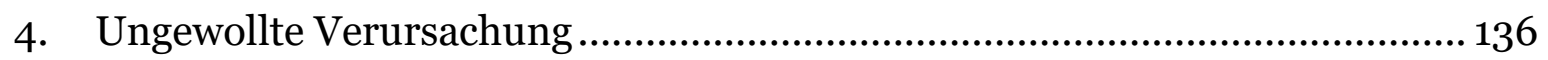

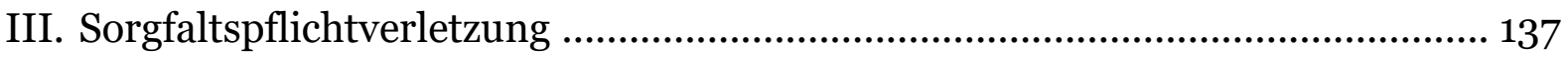

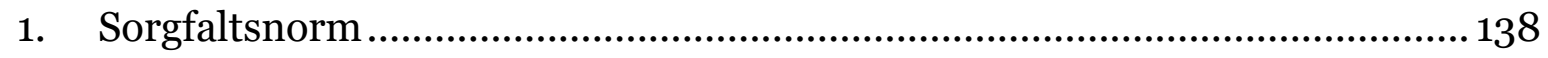

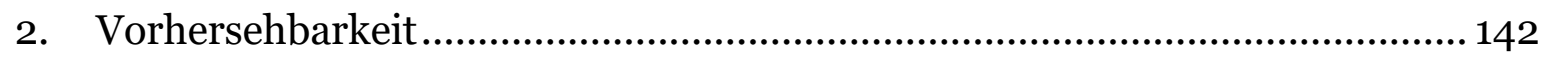

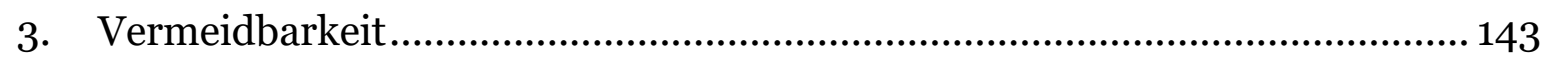

4. Erlaubtes Risiko ………………............................................................ 144

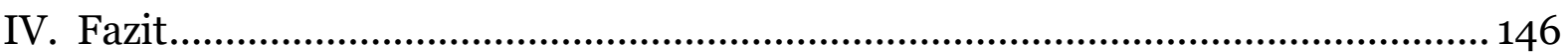

Zitiervorschlag: Marc Thommen/Martina Farag-Jaussi, Feuer und Flamme für Brandschutzvorschriften, in: sui-generis 2020, S. 132

URL: $\quad$ sui-generis.ch/126

DOI: $\quad$ https://doi.org/10.21257/sg.126

* Marc Thommen (marc.thommen@uzh.ch), Prof. Dr. iur. LL.M. (Cantab), ist Ordinarius für Strafrecht und Strafprozessrecht an der Universität Zürich. Martina Farag-Jaussi, MLaw, Rechtsanwältin, ist Doktorandin am Lehrstuhl von Prof. Thommen an der Universität Zürich. 


\section{Einleitung1}

1 Möbelpolitur-Fall: X. poliert ihre Möbel zuhause mit einem starken Putzmittel. Die darin getränkten Lappen sowie benutztes Haushaltspapier sammelt sie in einem Plastikeimer ohne Deckel, welchen sie auf dem Holzboden platziert. Das unbeaufsichtigte Material im Eimer entzündet sich von selbst und verursacht einen Brand mit erheblichen Schäden. $\mathrm{X}$. wird in zwei Instanzen wegen fahrlässiger Verursachung einer Feuersbrunst (Art. 222 StGB) verurteilt. Sie habe $\S 2$ des Brandschutzgesetzes des Kantons Aargau $^{2}$ verletzt, wonach jedermann im Umgang mit Wärme, Licht und anderen Energiearten die notwendige Vorsicht walten zu lassen hat, um Brände und Explosionen zu vermeiden. In einer summarischen Prüfung stuft das Bundesgericht das Verhalten von X. mangels Vorhersehbarkeit der Selbstentzündung als nicht fahrlässig ein. 3

2 Herdplatten-Fall: In der Wohnung von Y. ist während ihrer Abwesenheit der Schalter der Herdplatte aus ungeklärten Gründen auf Stufe sechs gedreht. Es kommt zu einem Brand mit massivem Sachschaden. Auf den Herdplatten sind die Überreste einer Fritteuse sowie von

1 Zu Akku-Brandfällen siehe Martina Farag-Jaussi, Strafrechtliche Folgen von brennenden Akkus, in: Nadine Zurkinden/Lukas Staffler/Julia Meier (Hrsg.), recht. innovativ, Analysen und Perspektiven von Assistierenden des RWI (APARIUZ), Band 21, zur Publikation vorgesehen im Mai 2020, S. $131 \mathrm{ff}$.

2 Gesetz über den vorbeugenden Brandschutz des Kantons Aargau (Brandschutzgesetz) vom 21. Februar 1989 (SAR 585.100).

3 Da X. nach Anhängigmachen der Beschwerde in Strafsachen verstorben ist, hat das Bundesgericht das Verfahren eingestellt und sich zu den Erfolgsaussichten lediglich in Zusammenhang mit den Entschädigungsansprüchen des Rechtsvertreters von $\mathrm{X}$. geäussert: Urteil des Bundesgerichts 6B_1091/2016 vom 18. Mai 2017 E. 1 und 2.
Textilien zu erkennen. Y. wird vom Bezirksgericht Laufenburg wegen fahrlässiger Verursachung einer Feuersbrunst verurteilt, weil sie Art. 3.2 Ziff. 1 der Brandschutzrichtlinie 12-15 der Vereinigung kantonaler Feuerversicherungen (VKF) 4 verletzt habe. Diese Bestimmung hält fest, dass Behälter mit brennbaren Gasen sowie andere brennbare Materialien von Kochherden so weit entfernt sein müssen, dass keine Brand- oder Explosionsgefahr entstehen kann. 5

3 Heizungs-Fall: Familie Z. bewohnt ein ehemaliges Bauernhaus mit Holzofenheizung. Infolge eines Überdrucks treten eines Abends Funken oder Flammen aus der (dafür vorgesehenen) Explosionsklappe aus, welche die um die Ecke gelagerten Zeitungen und damit den Heizungsraum in Brand setzen. Der Vater der Familie Z. wird mit Strafbefehl wegen fahrlässiger Verursachung einer Feuersbrunst verurteilt. ${ }^{6}$ Die Lagerung der brennbaren Materialen verletze Ziff. 7.3 Abs. 3 der (damals geltenden) Brandschutzrichtlinie betreffend wärmetech-

4 Vereinigung Kantonaler Feuerversicherungen (VKF), Brandschutzrichtlinie 12-15, Brandverhütung und organisatorischer Brandschutz, vom 1. Januar 2017.

5 Urteil des Bezirksgerichts Laufenburg ST.2016.31 vom 16. Juni 2016, welches lediglich mündlich begründet wurde und in der Zwischenzeit in Rechtskraft erwachsen ist. Im vorliegenden Beitrag wird mangels schriftlicher Begründung des Urteils auf den summarisch begründeten Strafbefehl vom 11. April 2016 sowie auf die mediale Berichterstattung in der Aargauer Online-Zeitung verwiesen (Marc Fischer, Fahrlässig Brand verursacht: Gericht reduziert Strafe für Hausbewohnerin, aargauerzeitung.ch vom 18. Juni 2016).

6 Der Strafbefehl vom 7. April 2003 konnte nicht erhältlich gemacht werden. Stattdessen wird im vorliegenden Beitrag auf den zivilrechtlichen Entscheid des Kreisgerichts Bern-Laupen vom 10. Juni 2005, Sammelstelle Gerichtsentscheide (SG) 2006 Nr. 1589, verwiesen, welcher immer wieder auf den Strafbefehl Bezug nimmt. 
nische Anlagen7, welche festhielt, dass leicht entzündbare Stoffe wie Holzwolle, Stroh oder Papier nicht im Heizraum gelagert werden dürfen. ${ }^{8}$

4 Brandgefahren sind ein realer Bestandteil unseres Alltags. Wir begegnen ihnen beim Kochen, beim Heizen und sogar bei der Möbelpolitur. Mit der Verwirklichung einer Brandgefahr drohen nicht nur grosse Schäden, sondern auch erhebliche rechtliche Konsequenzen für die Betroffenen. Anhand der eingangs geschilderten Fälle gibt der vorliegende Beitrag zunächst eine Übersicht über die Tatbestandsvoraussetzungen von Art. 222 StGB (II.). Anschliessend ist zu klären, ob die üblicherweise herangezogenen Brandschutzbestimmungen als strafrechtliche Sorgfaltsnormen taugen und welche Anforderungen an die Sorgfaltspflichten im privaten Bereich 9 zu stellen sind (III.). Der Beitrag schliesst mit einer Zusammenfassung (IV.).

\section{Verursachung}

\section{Tathandlung}

5 Den Tatbestand von Art. 222 StGB erfüllt, wer fahrlässig zum Schaden eines andern oder unter Herbeiführung einer Gemeingefahr eine Feuersbrunst verursacht (Abs. 1). Bringt der Täter fahrlässig Leib und Leben von Menschen in Gefahr, sind die Voraussetzungen des qualifizier-

7 Vereinigung Kantonaler Feuerversicherungen (VKF), Brandschutzrichtlinie 25-03, Wärmetechnische Anlagen, vom 26. März 2003 (nicht mehr in Kraft; vgl. nunmehr die Richtlinie vom 1. Januar 2017).

8 Entscheid des Kreisgerichts Bern-Laupen vom 10. Juni 2005, Sammelstelle Gerichtsentscheide (SG) 2006 Nr. 1589, Ziff. 21.

9 Der berufliche Umgang mit Brandgefahren - z.B. auf einer Baustelle - wird im vorliegenden Beitrag nicht berücksichtigt. ten Tatbestandes gemäss Abs. 2 erfüllt. Das Verhalten des Täters muss Ursache für den Erfolgseintritt sein und kann sowohl in einem Tun als auch - bei Vorliegen einer Garantenpflicht - in einer Unterlassung bestehen. ${ }^{10}$

6 Im Politur-Fall lag die Tathandlung (wohl) darin, dass die Beschuldigte X. das selbstentzündliche Material unbeaufsichtigt in einem Eimer ohne Deckel auf dem Holzboden hat stehen lassen. ${ }^{11} \mathrm{Im}$ Kochherd-Fall bestand das aktive Tun nach Ansicht des Gerichts darin, dass eine Fritteuse und Textilien auf dem Herd abgestellt wurden. ${ }^{12} \mathrm{Im}$ Heizungs-Fall wurde die Strafbarkeit am Lagern von Zeitungspapier in der Nähe der Explosionsklappe der Heizung angeknüpft.13

\section{Taterfolg}

7 Art. 222 StGB setzt zur Vollendung gleich wie die vorsätzliche Brandstiftung nach Art. 221 StGB - zwei verschiedene Erfolgseintritte voraus: Das Verursachen einer Feuersbrunst sowie entweder den Schaden eines anderen oder eine Gemeingefahr. Eine Feuersbrunst ist ein Feuer, welches ein solches Ausmass angenommen hat, dass es vom Urheber

$1 0 \longdiv { \text { Bruno Roelli in: Marcel Alexander Niggli/Hans } }$ Wiprächtiger (Hrsg.), Basler Kommentar Strafgesetzbuch (StGB), Strafrecht II, Art. 137-392 StGB, Jugendstrafgesetz, 4. Auflage, Basel 2018 (fortan zit.: BSK StGB II-Bearbeiter/in), Art. 222 N 6 mit Verweis auf Art. $221 \mathrm{~N} 9$.

11 Urteil des Bundesgerichts 6B_1091/2016 vom 18. Mai 2017, Sachverhalt und E. 3.2.3.

12 Strafbefehl der Staatsanwaltschaft RheinfeldenLaufenburg vom 11. April 2016, S. 2. Siehe auch Fischer (Fn. 5).

13 Entscheid des Kreisgerichts Bern-Laupen vom 10. Juni 2005, Sammelstelle Gerichtsentscheide (SG) 2006 Nr. 1589, Ziff. 19. 
nicht mehr beherrscht werden kann.14 Ein Schaden eines andern gemäss Art. 222 Abs. 1 StGB liegt vor, wenn eine fremde, d.h. nicht dem Täter gehörende Sache beschädigt wird. ${ }^{15}$ Die Versicherung als mittelbar Betroffene gilt nicht als geschädigt, wenn sie für einen Sachschaden im Eigentum des Täters aufkommen muss. ${ }^{16}$ Alternativ zum Erfordernis des Sachschadens kann der Tatbestand auch durch Herbeiführen einer Gemeingefahr für Sachen (Art. 222 Abs. 1 StGB) oder für Personen (Art. 222 Abs. 2 StGB) vollendet werden. Das Bundesgericht definiert die Gemeingefahr als Zustand, der die Verletzung von Rechtsgütern (Leib, Leben, Eigentum) in einem nicht zum Voraus bestimmten und abgegrenzten Umfang wahrscheinlich macht. ${ }^{17}$

8 Bei den in der Einleitung geschilderten drei Fällen waren die Erfolgsvoraussetzungen nicht oder nur am Rande Gegenstand der Erwägungen. Im Möbelpolitursowie im Herdplatten-Fall war ein Einsatz der Feuerwehr notwendig, was jeweils das Vorliegen einer Feuersbrunst indiziert. ${ }^{18}$ Im Heizungs-Fall platzte «wie

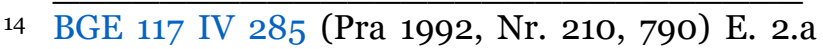
m.w.H.; ausführlich BSK StGB II-Roelli, Art. 222 $\mathrm{N} 6$ mit Verweis auf Art. $221 \mathrm{~N} 7$.

15 Nach h.L. wird nur der Sachschaden, nicht aber der Personenschaden von Abs. 1 erfasst: BSK StGB II-Roelli, Art. 222 N 6 mit Verweis auf Art. $221 \mathrm{~N} 11$ m.w.H.

16 BSK StGB II-Roelli, Art. 222 N 6 mit Verweis auf Art. $221 \mathrm{~N} 11$ m.w.H.

17 Zur Kritik an dieser Definition in der Lehre siehe Günter Stratenwerth/Felix Bommer, Schweizerisches Strafrecht, Besonderer Teil II, Straftaten gegen Gemeininteressen, 7. Auflage, Bern 2013, vor $\S 28 \mathrm{~N} 4$; siehe auch BSK StGB II-Roelli, vor Art. $221 \mathrm{~N} 11$ f. m.w.H.

18 Für den Möbelpolitur-Fall siehe die mediale Berichterstattung zum erstinstanzlichen Verfahren: Fritz Thut, «Burestobe»-Brand: Schuld waren selbstentzündbare Putzlappen, in: aargauerzeitung.ch vom 13. März 2015; für den Herdplatten- durch ein Wunder»19 eine unter Druck stehende Wasserleitung im Heizungsraum und löschte so das Feuer bis zu seiner Entdeckung am nächsten Morgen vollständig. Ob der Brand für den Urheber unbeherrschbar gewesen wäre, kann an dieser Stelle nicht abschliessend beurteilt werden.

9 Betreffend das zweite Erfolgserfordernis des Sachschadens bzw. der Gemeingefahr wurde im Möbelpolitur-Fall das Restaurant des Partners der Beschuldigten zerstört, ${ }^{20}$ womit ein Schaden eines anderen vorliegt. Gleiches gilt für den Heizungs-Fall, in welchem $\mathrm{Z}$. und seine Familie das beschädigte Gebäude zur Miete bewohnt haben. ${ }^{21}$ Im Herdplatten-Fall ist unklar, ob es sich bei der zerstörten Wohnung um Eigentum der Beschuldigten handelt oder nicht. Da die Wohnung jedoch Teil eines Sechsfamilienhauses war, ${ }^{22}$ ist davon auszugehen, dass zumindest eine Gemeingefahr für Sachen vorlag.

\section{Kausalität}

10 Beim fahrlässigen Erfolgsdelikt muss der Erfolg natürlich kausal durch das Verhalten des Täters verursacht worden sein, wobei als Ursache jede Handlung gilt, die nicht hinweg gedacht werden kann, ohne dass auch der Erfolg entfiele. ${ }^{23}$ Der Nach-

Fall siehe Strafbefehl vom 11. April 2016, S. 2 und Fischer (Fn. 5).

19 Entscheid des Kreisgerichts Bern-Laupen vom 10. Juni 2005, Sammelstelle Gerichtsentscheide (SG) 2006 Nr. 1589 Ziff. 11.

20 Thut (Fn. 18).

21 Entscheid des Kreisgerichts Bern-Laupen vom 10. Juni 2005, Sammelstelle Gerichtsentscheide (SG) 2006 Nr. 1589.

22 Fischer (Fn. 5).

23 Ausführlich zur natürlichen Kausalität siehe Marcel Alexander Niggli/Stefan Maeder in: Marcel Alexander Niggli/Hans Wiprächtiger (Hrsg.), Basler Kommentar Strafgesetzbuch 
weis der Kausalität kann bei Art. 222 StGB in der Praxis vor allem dann Schwierigkeiten bereiten, wenn die Brandursache nicht vollständig geklärt werden kann. ${ }^{24}$

Oft lassen sich die Strafverfolgungsbehörden davon jedoch nicht beirren und bejahen die natürliche Kausalität mit pauschalen Behauptungen. Im HeizungsFall, wo der Untersuchungsrichter es im Strafbefehl für möglich hielt, dass 1) eine Kartonschachtel durch noch nicht erkaltete Asche oder 2) durch Funkenwurf aus der Explosionsklappe oder dass 3) die um die Ecke gelagerten Zeitungen durch Funkenwurf aus der Explosionsklappe in Brand gesetzt wurden,25 vermag dies noch halbwegs zu überzeugen. Immerhin entsprechen alle drei möglichen Abläufe dem vorgeworfenen Verhalten, dass brennbares Material im Heizraum gelagert worden ist. Im Herdplatten-Fall demgegenüber hat das zuständige Bezirksgericht festgehalten, dass sowohl offenbleiben könne, wer den Drehschalter des Kochherdes betätigt habe (die Beschuldigte selbst mit Jacke oder Tasche oder deren Hunde) als auch wo und in welcher Entfernung zur Herdplatte sich die gefundenen Gegenstände genau befunden hätten. Damit blieben wesentliche Elemente des Sachverhaltes ungeklärt. Der Verteidiger der Beschuldigten

(StGB), Strafrecht I, Art. 1-392 StGB, Jugendstrafgesetz, 4. Auflage, Basel 2019 (fortan zit.: BSK StGB I-Bearbeiter/in), Art. $12 \mathrm{~N} 90 \mathrm{ff}$.

24 So etwa in einem Urteil des Kreisgerichts Wil ST.2019.25 vom 16. Juli 2019; über das rechtskräftige Urteil, welches unbegründet geblieben ist, wurde in der Republik online vom 7. August 2019 berichtet: Sina Bühler, Brandgefährlicher Akku.

25 Entscheid des Kreisgerichts Bern-Laupen vom 10. Juni 2005, Sammelstelle Gerichtsentscheide (SG) 2006 Nr. 1589 Ziff. 11 mit einer Zusammenfassung der Ausführungen im Strafbefehl. zweifelte den Kausalzusammenhang mit dem berechtigten Einwand an, dass ein Lappen doch ganz verbrannt wäre, wenn er das Feuer ausgelöst hätte. ${ }^{26}$ Im Möbelpolitur-Fall demgegenüber war die Brandursache unbestritten und der natürliche Kausalzusammenhang wohl erstellt: Hätte die Beschuldigte nicht das selbstentzündliche Material unbeaufsichtigt in einen Eimer ohne Deckel auf den Holzboden gestellt, wäre es nicht zum Erfolgseintritt i.S.v. Art. 222 StGB gekommen. ${ }^{27}$

\section{Ungewollte Verursachung}

12 Gemäss Art. 12 Abs. 3 Satz 1 StGB begeht ein Verbrechen oder Vergehen fahrlässig, wer die Folge seines Verhaltens aus pflichtwidriger Unvorsichtigkeit nicht bedenkt oder darauf nicht Rücksicht nimmt. Fahrlässiges Verursachen einer Feuersbrunst bedeutet, dass bereits das Entfachen des Feuers oder spätestens aber die Taterfolge (Feuersbrunst, Gemeingefahr, Schaden) ungewollt sind, d.h. auch nicht in Kauf genommen wurden. Mit dem Wegfallen des voluntativen Elements entfällt der Vorsatz. Ein Vorsatzdelikt stand bei keinem der drei Beispielfälle zur Diskussion.

13 Auf der kognitiven Seite (Wissen) wird beim Fahrlässigkeitsdelikt danach unterschieden, ob der Täter den Erfolgseintritt für möglich gehalten, aber auf dessen Ausbleiben vertraut hat (bewusste Fahrlässigkeit) oder ob er den Erfolgseintritt überhaupt nicht bedacht hat (unbewuss-

26 Fischer (Fn. 5). Mangels schriftlicher Urteilsbegründung bleibt Spekulation, ob der Kausalzusammenhang erstellt war.

27 Dem Urteil des Bundesgerichts 6B_1091/2016 vom 18. Mai 2017 sind keine Erwägungen zum Kausalzusammenhang zu entnehmen. 
te Fahrlässigkeit). ${ }^{28}$ Die Frage nach der Voraussicht ist tatsächlicher Natur (Wissen) und klar von der normativ zu beurteilenden Vorhersehbarkeit zu trennen (Wissenmüssen), welche erst auf der Ebene der Sorgfaltspflichtverletzung relevant wird (III.2.).

14 Im Politur-Fall ging es um unbewusste Fahrlässigkeit. Der Betroffenen war (wohl) ${ }^{29}$ nicht klar, dass sich Putzmittel spontan entzünden können. ${ }^{30}$

15 Das Urteil zum Heizungs-Fall äussert sich nicht bezüglich der Klassifizierung der Fahrlässigkeit: Bewusste Fahrlässigkeit läge vor, wenn der Mieter die Gefahr für möglich hielt, dass Funken aus der Explosionsklappe die um die Ecke gelagerten Zeitungen und damit den Heizungsraum in Brand setzen konnten, er aber darauf vertraute, dass dies nicht eintreten werde. Unbewusste Fahrlässigkeit läge vor, wenn Z. die Möglichkeit eines so verursachten Brandes nicht bedachte.

Auch im Herdplatten-Fall wurde die Frage nach der Art der Fahrlässigkeit nicht beantwortet. Erkannte die Beschuldigte, dass durch das Lagern von Gegenständen in der Nähe der Herdplatten ein Brand entstehen kann, läge bewusste Fahrläs-

28 BGE 136 IV 76 E. 2.3.1.

29 Eine explizite Tatsachenfeststellung zum tatsächlichen Wissen der Betroffen findet sich im Bundesgerichtsurteil nicht, es wird lediglich festgehalten, dass «die Beschwerdeführerin nicht mit einer Selbstentzündung des Mittels rechnen musste», mithin, was sie hätte wissen müssen oder nicht (Urteil des Bundesgerichts 6B_1091/2016 vom 18. Mai 2017 E. 3.2.3). Für unbewusste Fahrlässigkeit spricht die Berichterstattung zum erstinstanzlichen Verfahren, siehe Thut (Fn. 18).

30 Urteil des Bundesgerichts 6B_1091/2016 vom 18. Mai 2017 E. 3.2.3; siehe auch III.2. sigkeit vor, bedachte sie diese Möglichkeit nicht, unbewusste Fahrlässigkeit. Daran schliesst unweigerlich die Frage an, woraus sich ergibt, dass die Verkennung der Feuergefahr bzw. das Vertrauen auf deren Ausbleiben pflichtwidrig war.

\section{Sorgfaltspflichtverletzung}

17 Art. 12 Abs. 3 Satz 2 StGB definiert eine Unvorsichtigkeit (imprévoyance, imprevidenza) als pflichtwidrig (coupable, colpevole), wenn der Täter die Vorsicht nicht beachtet, zu der er nach den Umständen und nach seinen persönlichen Verhältnissen verpflichtet ist. In den Worten des Bundesgerichts ist die Unvorsichtigkeit pflichtwidrig, «wenn der Täter zum Zeitpunkt der Handlung aufgrund seiner Kenntnisse und Fähigkeiten die damit bewirkte Gefährdung [...] hätte erkennen können und wenn er zugleich die Grenzen des erlaubten Risikos überschritten hat. Der bewusst fahrlässig handelnde Täter weiss um das Risiko der Tatbestandsverwirklichung, vertraut aber aus pflichtwidriger Unvorsichtigkeit darauf, dass der von ihm als möglich vorausgesehene Erfolg nicht eintreten werde» 3 .

18 Der zweite Satz bedarf der Präzisierung: Bei der bewussten Fahrlässigkeit wird der Erfolgseintritt als möglich antizipiert. Es liegt somit nicht Unvorsicht im Wortsinne vor. Dem Täter wird hier vorgeworfen, dass er nicht auf das Ausbleiben des (vorhergesehenen) Erfolgs vertrauen durfte. Er hat sich zu wenig Sorgen um die Rechtsgutsverletzung gemacht. Sein Verhalten ist insoweit sorglos oder eben unsorgfältig. Nur bei der unbewussten Fahrlässigkeit handelt der Täter sprich-

31 BGE 136 IV 79 E. 2.3.1. m.w.H. 
wörtlich unvorsichtig. Er sieht die drohende Rechtsgutsverletzung nicht und diese Unvorsicht wird ihm vorgeworfen. In der Sache besteht aber Einigkeit, dass sowohl die Unsorgfalt als auch die Unvorsicht pflichtwidrig sein müssen.

In der Schweiz ${ }^{2}$ bislang nicht vertieft diskutiert ist die Frage, inwiefern die inhaltsleere Umschreibung der Sorgfaltspflichtverletzung in Art. 12 Abs. 3 StGB mit dem Legalitätsprinzip, insbesondere dem Bestimmtheitsgebot, in Einklang zu bringen ist.33 Einerseits verlangt die Rechtsprechung, dass Strafnormen so präzise formuliert sind, dass der Bürger sein Verhalten danach richten kann. Andererseits könne der Gesetzgeber aber auch nicht darauf verzichten, vage Begriffe zu verwenden, deren Auslegung der Praxis überlassen werden müsse. 34 Möglicherweise ist die Rechtsprechung bei den Fahrlässigkeitsdelikten mit dem Bestimmtheitsgebot deshalb etwas kulanter, weil in aller Regel weniger gravierende Rechtsfolgen drohen. 35

Ausgangspunkt der nach den Umständen zu beachtenden Vorsicht/Sorgfalt ist in der Regel eine Sorgfaltsnorm (1.). Sorgfaltspflichtwidrig ist nur die Verursachung vorhersehbarer (2.) und vermeid-

Für Deutschland siehe Gunnar Duttge, Zur Bestimmtheit des Handlungsunwerts von Fahrlässigkeitsdelikten, Habil. Bochum 1999, Tübingen 2001, passim.

33 Die Zweitautorin geht dieser Grundsatzfrage im Rahmen ihres Dissertationsprojekts nach. Sie untersucht dabei ebenfalls, ob bzw. unter welchen Voraussetzungen der Beizug von Sorgfaltsnormen zur Bestimmung der Sorgfaltspflichtverletzung möglich sein soll.

34 BGE 138 IV 13 E. 4.1.

35 Für praktisch gewichtige Ausnahmen im Nebenstrafrecht vgl. Marc Thommen, Art. 333 Abs. 7 StGB, Grenzenlose Fahrlässigkeit im Nebenstrafrecht?, recht 2013, Heft 6, 276 ff., 287. barer (3.) Erfolge, und dies auch nur, wenn das Verhalten jenseits des erlaubten Risikos liegt (4.).

\section{Sorgfaltsnorm}

21 Sorgfaltsnormen sind aus leidvoller Erfahrung geronnene Handlungsanleitungen. «Wo besondere, der Unfallverhütung und der Sicherheit dienende Normen ein bestimmtes Verhalten gebieten, bestimmt sich das Mass der zu beachtenden Sorgfalt in erster Linie nach diesen Vorschriften [...].»36 Der Vorwurf der Fahrlässigkeit kann gemäss Bundesgericht aber auch auf allgemeine Rechtsgrundsätze wie etwa den Gefahrensatz gestützt werden. Danach hat jeder, der eine Gefahr schafft, alles Zumutbare vorzukehren, um zu verhindern, dass die Gefahr sich realisiert. Andernfalls hat er die Tätigkeit ganz zu unterlassen. 37

\section{a) Quelle}

22 Besondere Sorgfaltsnormen können aus unterschiedlichen Quellen stammen: Von eidgenössischen oder kantonalen Gesetzen und Verordnungen über Empfehlungen staatlicher Stellen bis hin zu privaten Regelwerken..$^{8}$ Für die fahrlässige Verursachung einer Feuersbrunst im privaten Bereich39 können als Quellen von Sorgfaltsnormen kantonale Feuer- und Brandschutzgesetze, die Brandschutzvorschriften der Vereinigung kantonaler

36 BGE 143 IV 138 E. 2.1.

37 Urteil des Bundesgerichts 6B_1411/2017 vom 23. Mai 2018 E. 2.1.

38 BSK StGB I-Niggli/Mäder, Art. 12 N 111.

39 Für den beruflichen Umgang mit Brandgefahren - z.B. auf einer Baustelle - gelten erstens detailliertere Brandschutzvorschriften der VKF; zweitens gibt es zahlreiche berufsspezifische Vorschriften, welche wir in diesem Beitrag nicht berücksichtigen (z.B. EKAS Richtlinie Nr. 1825, Brennbare Flüssigkeiten, Lagern und Umgang, Ausgabe Mai 2005). 
Feuerversicherungen (VKF) sowie Herstellerangaben $\mathrm{zu}$ brandgefährlichen Produkten diskutiert werden.

23 Aufgrund ihrer demokratischen Legitimation eignen sich Bestimmungen der kantonalen Feuerpolizei- und Brandschutzgesetzgebung grundsätzlich am besten als Rechtsquellen von Sorgfaltsnormen. Soweit es um Brände ausserhalb des beruflichen Bereichs geht, ist jedoch darauf zu achten, dass sich die Vorschriften an Privatpersonen richten (z.B.: «Jedermann ist verpflichtet, alles ihm $\mathrm{Zu}$ mutbare vorzukehren, um Brand- und Explosionsschäden zu verhindern»40).

24 Nach der Rechtsprechung sollen auch die Brandschutzvorschriften der Vereinigung kantonaler Feuerversicherungen (VKF) als Sorgfaltsnormen im privaten Bereich dienen können. ${ }^{41}$ Diese bestehen aus einer Brandschutznorm 1-15 und den Brandschutzrichtlinien 10-15 bis 28-15.42 Das Interkantonale Organ Technischer Handelshemmnisse (IOTH) hat die VKF im Jahr 2010 mit der Ausarbeitung der Brandschutzvorschriften beauftragt und per 1. Januar 2015 in Kraft gesetzt. Sie sind seither in der ganzen Schweiz ver-

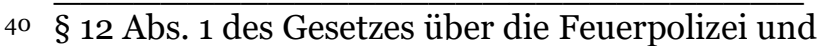
das Feuerwehrwesen des Kantons Zürich vom 24. September 1978 (LS 861.1), unter der Marginalie «Pflichten Privater».

41 Urteil des Bundesgerichts 6B_948/2010 vom 12. Mai 2011 E. 3.1.2.; in der mündlichen Urteilsbegründung im Herdplatten-Fall hat das Bezirksgericht Laufenburg festgehalten, dass die einschlägige Brandschutzrichtlinie sinngemäss auch auf Private angewandt werden könne, zumal sie auf allgemeiner Lebenserfahrung beruhe, siehe Fischer (Fn. 5). Die Brandschutzvorschriften stellen in erster Linie Anforderungen an den Brandschutz in Betrieben und Gebäuden und richten sich nur sehr beschränkt an Privatpersonen. Allgemeine Sorgfaltspflichten finden sich in der Brandschutznorm 1-15, Bst. B Art. 17 ff. sowie in der Brandschutzrichtlinie 12-15, Ziff. 3.

42 Die Brandschutzvorschriften der VKF sind online. bindlich.43 Bezüglich der Eignung von Brandschutzvorschriften der VKF als Quellen strafrechtlicher Sorgfaltsnormen sind zwei allgemeine Vorbehalte anzubringen. Erstens weisen sie im Vergleich zu den Brandschutzgesetzen eine deutlich geringere demokratische Legitimation auf.44 Zweitens gibt es ein Befangenheitsproblem: Nach eigener Darstellung besteht die Aufgabe der Vereinigung Kantonaler Feuerversicherungen (VKF) darin, ihre Mitglieder im Bereich Prävention $\mathrm{zu}$ unterstützen: «Generell richtet die VKF ihren Fokus auf die Verminderung von Personen- und Gebäudeschäden. Demzufolge entwickelt die VKF im Auftrag der Kantonalen Gebäudeversicherungen Instrumente zur Minimierung derartiger Schäden.»45 Die VKF ist mit anderen Worten eine Vereinigung, deren primäre Aufgabe darin besteht, die ihr angeschlossenen Versicherungen vor Schadenszahlungen zu bewahren. Die Brandschutzvorschriften wurden somit von einer Organisation ausgearbeitet, welche ebenso wie ihre Mitglieder ein manifestes wirtschaftliches Interesse an der Vermeidung von Bränden hat. Diese einseitige Interessenlage vermag auch dadurch nicht beseitigt zu werden, dass die Richtlinien formell durch das IOTH

43 Beschluss des Interkantonalen Organs Technische Handelshemnisse (IOTH) vom 18. September 2014, z.B. in der Gesetzessammlung des Kantons Aargau (SAR 585.115).

44 Dies gilt a fortiori für rein private Regelwerke, wie etwa die vom Bundesgericht ebenfalls herbeigezogenen FIS-Regeln, welche im Gegensatz zu den Brandschutzrichtlinien nicht durch ein interkantonales Organ, sondern durch einen privatrechtlichen Verein erlassen wurden (vgl. BGE 106 IV 350 E. 3a).

45 Homepage der Vereinigung kantonaler Gebäudeversicherungen in der Rubrik Organisationen, VKF - Vereinigung Kantonaler Feuerversicherungen. 
in Kraft gesetzt wurden. ${ }^{6}$ Nun könnte man argumentieren, dass ein wirtschaftliches Interesse der beste Garant für effektiven Brandschutz ist. Wegen der Brandschutzvorschriften verzeichne die Schweiz - nach Singapur - weltweit am wenigsten Brandtote. 47 Dass die Vorschriften geeignet sind, Polizeigüter zu schützen und Haftungsrisiken einzudämmen, wird nicht in Abrede gestellt. Im Gegenteil, das tun sie höchst effizient. Sie führen jedoch zu einer sehr unilateralen Schadensallokation zu Gunsten der Versicherer und zu Lasten der Privaten. Man könnte deshalb bereits für die zivilrechtliche, mit Sicherheit aber für die strafrechtliche Haftung kritisch hinterfragen, ob diese Schadensverteilung gerecht ist und ob Brandschutzvorschriften der VKF als Quellen von Sorgfaltsnormen in Frage kommen sollen.

Offensichtlich nicht als Quellen von Sorgfaltsnormen eignen sich Herstellerangaben zu brandgefährlichen Produkten wie aufgedruckte Warnhinweise oder Bedienungsanleitungen. Sie dienen typischerweise dem Haftungsausschluss der Hersteller und kommen als Grundlage für die strafrechtlichen Sorgfaltspflichten im privaten Bereich nicht in Betracht. ${ }^{8}$ Ist erstellt, dass eine Person Kenntnis von den Herstellerangaben hatte, wirkt sich dies auf die Art der Fahrlässigkeit (be-

$46 \overline{\text { So das Argument eines Tagungsteilnehmers (vgl. }}$. Titelfussnote oben).

47 So die Vereinigung kantonaler Gebäudeversicherungen, Rubrik Ziele \& Aufgaben, wirksamer Brandschutz.

48 Vgl. die (divergierenden) Hinweise zum Aufladen für die Hoverboards "City Blitz®», S. 12 ff. und «Denver DBO-6550 MK2», Kapitel 6 sowie «Rusta», S. 46 ff.; zur Brandgefahr von Akkus in Hoverboards siehe (mündlich begründetes) Urteil des Kreisgerichts Wil ST.2019.25 vom 16. Juli 2019, über welches online berichtet wurde (Bühler, Fn. 1); siehe auch Farag-Jaussi (Fn. 1), S. 136. wusst oder unbewusst) sowie allenfalls auf die subjektive Konkretisierung der Sorgfaltspflicht (besondere Kenntnisse) aus.

\section{b) Mindestanforderungen}

26 Die Sorgfaltsnorm ist bei der Beurteilung der Sorgfaltspflichtverletzung lediglich der Ausgangspunkt.49 Sie ist sozusagen das «Rohmaterial»50, aus dem die konkrete Sorgfaltspflicht gewonnen wird. Als Mindestanforderung muss eine Bestimmung über eine reine Erfolgs- oder Gefahrenverhinderungspflicht hinausgehen, um als Sorgfaltsnorm gelten zu können. ${ }^{11}$ Die pauschal formulierten Bestimmungen in den kantonalen Feuer- und Brandschutzgesetzen sowie in den Brandschutzvorschriften der VKF, welche sich an Privatpersonen richten, erfüllen oft bereits diese Mindestanforderung an strafrechtliche Sorgfaltsnormen nicht. ${ }^{2}$

27 Im Möbelpolitur-Fall etwa stufte das Bundesgericht $\S 2$ Abs. 1 des aargauischen Brandschutzgesetzes53 deshalb zu Unrecht als genügende Grundlage für die Sorgfaltspflichtverletzung ein.54 Danach

49 BGE 135 IV 56 E. 2.1.

50 Andreas Donatsch, Sorgfaltsbemessung und Erfolg beim Fahrlässigkeitsdelikt, Habil. Zürich 1987, S. 114 .

51 Z.R. weist Günter Stratenwerth darauf hin, dass strafrechtlich sanktionierte Sorgfaltspflichten nicht gebieten können, bestimmte Erfolge als solche zu verhindern, sondern nur, die zu ihnen führenden Geschehensabläufe (Schweizerisches Strafrecht, Allgemeiner Teil I: Die Straftat, 4. Auflage, Bern 2011, § $16 \mathrm{~N}$ 9).

52 In allgemeiner Weise zweifelnd mit Bezug auf das aus nullum crimen sine lege abgeleitete Gebot der Tatbestandsbestimmtheit Magdalena Rutz, der objektive Tatbestand des Fahrlässigkeitsdelikts, ZStrR 89/1973, S. 358, 360.

53 Gesetz über den vorbeugenden Brandschutz des Kantons Aargau (Brandschutzgesetz) vom 21. Februar 1989 (SAR 585.100).

54 Urteil des Bundesgerichts 6B_1091/2016 vom 18. Mai 2017 E. 3.2.2. 
hat «[j]edermann [...] im Umgang mit Wärme, Licht und anderen Energiearten die notwendige Vorsicht walten zu lassen, um Brände und Explosionen zu vermeiden [...].» Diese Bestimmung stellt eine reine Erfolgsverhinderungspflicht dar, indem sie verlangt, dass Brände und Explosionen zu vermeiden sind. Sie ist als Handlungsanleitung unbrauchbar, weil offenbleibt, wie das Feuer verhindert werden könnte.

Im Herdplatten-Fall hat die Beschuldigte nach Ansicht des Bezirksgerichts Art. 3.2 Ziff. 1 der Brandschutzrichtlinie 12-15 verletzt, wonach brennbare Materialien von Kochherden so weit entfernt sein müssen, dass keine Brand- oder Explosionsgefahr entstehen kann. Das Bezirksgericht verkennt dabei, dass die Bestimmung keinen Hinweis darauf enthält, wie weit Fritteusen von Kochherden entfernt sein müssen und - mit Blick auf den Luftabzug - sein dürfen. Auch sie statuiert somit eine blosse und deshalb unbrauchbare Gefahrenverhinderungspflicht.55

Im Heizungs-Fall demgegenüber kam eine konkrete, verständliche Sorgfaltsnorm zur Anwendung. Das Gericht ging davon aus, dass die Lagerung der brennbaren Materialien Ziff. 7.3 Abs. 3 der (damals geltenden) Brandschutzrichtlinie betreffend wärmetechnische Anlagen $^{56}$ verletzte, welche festhielt, dass leicht entzündbare Stoffe wie Holzwolle,

55 Allzu weit kann die Entfernung nicht sein, weil gerade offene Fritteusen ebenfalls unter der Entlüftungsanlage, welche oberhalb des Herdes ist, stehen müssen.

56 Vereinigung Kantonaler Feuerversicherungen (VKF), Brandschutzrichtlinie 25-03, Wärmetechnische Anlagen, vom 26. März 2003 (nicht mehr in Kraft).
Stroh oder Papier nicht im Heizraum gelagert werden dürfen. 57

\section{c) Anpassung an den Einzelfall}

3o Selbst wenn im Einzelfall eine konkrete Sorgfaltsnorm vorliegt, die keine blosse Erfolgsvermeidung postuliert, kann von deren Verletzung nicht automatisch auf die Verletzung einer Sorgfaltspflicht geschlossen werden. Wie das Bundesgericht festhält, «begründet nicht jeder Verstoss gegen eine [...] Verhaltensnorm den Vorwurf der Fahrlässigkeit [...]. Die Vorsicht, zu der ein Täter verpflichtet ist, wird letztlich durch die konkreten Umstände und seine persönlichen Verhältnisse bestimmt.»58 Dabei muss gelten, dass die Anforderungen im Einzelfall umso höher sind, je offener und allgemeiner die Sorgfaltsnorm formuliert ist. In dieser Konkretisierung der Sorgfaltspflicht kommt die Konzeption des Fahrlässigkeitsunrechts zum Ausdruck, welche Art. 12 Abs. 3 StGB zugrunde liegt. Nach wohl herrschendem schweizerischen Verständnis gilt ein situationsbezogener, individualisierter Sorgfaltsmassstab.59 Es kommt auf alle relevanten Umstände sowie die individuellen Fähigkeiten der beschuldigten Person an. ${ }^{60}$ Situationsbezo-

$57 \overline{\text { Entscheid des Kreisgerichts Bern-Laupen vom }}$ 10. Juni 2005, Sammelstelle Gerichtsentscheide (SG) 2006 Nr. 1589, Ziff. 21.

58 BGE 135 IV 56 E. 2.1.; Donatsch (Fn. 50), S. 124 f.

59 Anders z.B. in Deutschland, wonach gemäss h.L. eine gewissenhafte Durchschnittsperson des Amts-, Berufs- oder Gewerbekreises des Täters massgebend ist, siehe Detlev Sternberg-Lieben/ Frank Schuster, in: Schönke/Schröder Strafgesetzbuch Kommentar, 29. Auflage, München 2014, § 15, N 135 m.N.

60 BGE 97 IV 169 E. 2; anders noch BGE 86 IV 153 E. 1, wo ein rein objektiver Massstab relevant war. In der Lehre variiert jedoch der Grad der Individualisierung: Teilweise wird verlangt, die tatsächlichen individuellen Verhältnisse umfassend zu berücksichtigen (siehe etwa Kurt Seelmann/ Christopher Geth, Strafrecht Allgemeiner Teil, 
gen sind diejenigen Faktoren zu berücksichtigten, welche sich auf die Höhe der Brandgefahr auswirken (z.B. das Wetter). ${ }^{61} \mathrm{Zu}$ den individuellen Fähigkeiten gehören unter anderem die «geistigen Anlagen, die Bildung und die berufliche Erfahrung».62 Die Pflichtwidrigkeit kann jedoch auch darin liegen, dass die Aufgabe überhaupt übernommen wurde, ohne über die erforderlichen Fähigkeiten zu verfügen (Übernahmefahrlässigkeit). ${ }^{6}$ Umgekehrt können besondere Kenntnisse und Fähigkeiten zu einem strengeren Sorgfaltsmassstab führen. ${ }^{64}$

Klar abzulehnen ist daher das Urteil im Herdplatten-Fall, in welchem das Bezirksgericht im Einklang mit der Staatsanwaltschaft alleine vom Verstoss gegen Art. 3.2 Ziff. 1 der Brandschutzrichtlinie $12-15^{65}$ auf eine Sorgfaltspflichtwidrigkeit

6. Auflage, Basel 2016, N 467 f.; BSK StGB INiggli/Maeder, Art. 12 N 99 ff.; 111 ff.; Marc JeanRichard-dit-Bressel/Stefan Trechsel, in: Stefan Trechsel/Mark Pieth(Hrsg.), Praxiskommentar Schweizerisches Strafgesetzbuch, 3. Auflage, Zürich 2017 (fortan zit. PK StGB-Bearbeiter/in), Art. 12 $\mathrm{N} 31$, 35); teilweise werden die persönlichen Verhältnisse nur in verallgemeinerter Form berücksichtigt, indem z.B. für verschiedene Alters-, Bildungs- und Berufsgruppen verschiedene Massstäbe angelegt werden, letztlich aber gleich wie in der deutschen Lehre eine Durchschnittsperson massgebend sein soll (siehe etwa Hans Walder, Probleme bei Fahrlässigkeitsdelikten, in: ZBJV 104/1968, S. 161-189, 170; Martin Killias et al., Grundriss des Allgemeinen Teils des Schweizerischen Strafgesetzbuchs, 2. Auflage, Bern 2017, $\mathrm{N}$ 330; Bernard Corboz in: Alain Macaluso et al. (Hrsg.), Commentaire Romand, Code pénal I, Art. 1-110 StGB, Basel 2017, Art. 12 N 151 f.).

61 PK StGB-Jean-Richard-dit-Bressel/Trechsel, Art. 12 N 31.

62 BGE 97 IV 169 E. 2.

63 Urteil des Bundesgerichts 6B_174/2013 vom 20. Juni 2013 E. 3.3.4; Urteil des Bundesgerichts 6B_1049/2015 vom 6. September 2016 E. 2.5.

64 BGE 97 IV 169 E. 2.

65 Was aus verschiedenen Gründen schon gar nicht als strafrechtliche Sorgfaltsnorm taugt, siehe III.1. a)-b). geschlossen hat. ${ }^{66}$ Auch im Heizungs-Fall wurde, soweit ersichtlich, darauf verzichtet, nach der Feststellung der Verletzung der Brandschutzrichtlinie eine weitere Konkretisierung der Sorgfaltspflicht vorzunehmen. ${ }^{67}$ Hier hätte festgelegt werden müssen, welche Brennmaterialien in welchem Abstand zum Ofen wie gelagert werden dürfen. Im Möbelpolitur-Fall hat das Bundesgericht demgegenüber eine Konkretisierung vorgenommen und die Sorgfaltspflichtverletzung am Erfordernis der Vorhersehbarkeit scheitern lassen.

\section{Vorhersehbarkeit}

32 Von einer pflichtwidrigen (coupable, colpevole) Unvorsicht bzw. Unsorgfalt kann nur die Rede sein, wenn der Erfolgseintritt für den Täter vorhersehbar war. Jenseits des Vorhersehbaren kann kein Vorwurf erhoben werden. ${ }^{68}$ Die normativ zu bestimmende Vorhersehbarkeit sollte nicht mit der tatsächlichen Voraussicht verwechselt werden:69 Die Vorhersehbarkeit ist aus einer ex-ante-Perspektive zu beurteilen, d.h. es ist danach zu fragen, was der Täter zum Zeitpunkt der Tat aufgrund der Umstände sowie seiner Kenntnisse und Fähigkeiten hätte erkennen müssen. ${ }^{70}$ Diese Betrachtungsweise funktioniert jedoch in der Theorie besser als in der Praxis. Im Rückblick wird die Vor-

$66 \overline{\text { Urteilsdispositiv des Bezirksgerichts Laufenburg }}$ vom 16. Juni 2016 mit Verweis auf den Strafbefehl vom 11. April 2016; siehe auch Fischer (Fn. 5).

67 Entscheid des Kreisgerichts Bern-Laupen vom 10. Juni 2005, Sammelstelle Gerichtsentscheide (SG) 2006 Nr. 1589 Ziff. 21.

68 Siehe auch Stratenwerth (Fn. 51), AT I4, § 16 N 8.

69 Bei der Voraussicht geht es um die Frage, was ein Täter tatsächlich vorausgesehen hat. Sie legt nur fest, ob bewusste oder unbewusste Fahrlässigkeit vorliegt (siehe dazu auch III.).

70 BGE 135 IV 56 E. 1.2. 
hersehbarkeit nämlich oftmals überschätzt, da im Zeitpunkt der gerichtlichen Beurteilung zwangsläufig bereits etwas passiert ist (sog. Rückschaufehler oder «hindsight bias»). ${ }^{71}$ Bei der Vorhersehbarkeit wendet das Bundesgericht im Normalfall den Massstab der Adäquanz an, d.h. es ist danach zu fragen, ob nach dem gewöhnlichen Lauf der Dinge und der allgemeinen Lebenserfahrung mit einem Erfolg der eingetretenen Art zu rechnen war. ${ }^{72}$ Verfügt die beschuldigte Person jedoch über besondere Fähigkeiten oder fehlen ihr solche, beurteilt es die Vorhersehbarkeit nach einem individualisierten Sorgfaltsmassstab,73 der auch sonst für die Beurteilung der Sorgfaltspflichtverletzung Anwendung findet.74

Im Herdplatten-Fall haben sich die Strafbehörden - soweit ersichtlich nicht mit der Vorhersehbarkeit auseinandergesetzt, sondern diese einfach implizit mit dem Schuldspruch bejaht. Im Heizungs-Fall hat sich immerhin das Zivilgericht summarisch zur adäquaten Kausalität geäussert.75 Nicht vorhergesehen werden musste die Brandgefahr im Möbelpolitur-Fall, weil die Selbstentzündlichkeit vom Bundesgericht z.R. als unvorhersehbar eingestuft wurde. Als Serviceangestellte habe die Beschwerdeführerin über kein besonderes Wissen um chemische Zusammenhänge verfügt und deshalb nicht mit einer Selbstent-

71 Zum Ganzen siehe Roman Elsener, «Das hätte man wissen müssen!», in: sui-generis 2015, S. 117, 118.

72 Statt vieler BGE 135 IV 56 E. 2.1. Kritisch dazu BSK StGB I-Niggli/Maeder, Art. 12 N 97.

73 BGE 97 IV 169 E. 2.

74 Siehe dazu soeben III.1.c).

75 Entscheid des Kreisgerichts Bern-Laupen vom 10. Juni 2005, Sammelstelle Gerichtsentscheide (SG) 2006 Nr. 1589, Ziff. 19. zündung der mit der Politur getränkten Lappen rechnen müssen. ${ }^{7}$

34 Die Vorhersehbarkeit ist sowohl bei der bewussten als auch bei der unbewussten Fahrlässigkeit zu prüfen. Bei der unbewussten Fahrlässigkeit stellt sich die Frage, ob der in concreto nicht vorhergesehene Erfolg hätte vorhergesehen werden müssen. Bei der bewussten Fahrlässigkeit, also wenn die Gefahr gesehen, aber darauf vertraut wurde, dass sie sich nicht verwirklicht, liegt in aller Regel auch Vorhersehbarkeit vor. Wer bei trockenem Hochsommerwetter vorhersieht, dass er mit Feuerwerkskörpern eine Feuersbrunst entfachen könnte und nur darauf vertraut, dass nichts passieren werde, dem wird man die Vorhersehbarkeit der Folgen angesichts der klimatischen Umstände problemlos nachweisen können. Dennoch darf nicht unbesehen von der tatsächlichen Voraussicht auf die normativ zu bestimmende Vorhersehbarkeit geschlossen werden. Hätte eine extrem gewissenhafte Person im MöbelpoliturFall die Brandgefahr tatsächlich vorausgesehen, hätte das nichts an der generellen Unvorhersehbarkeit geändert. Andernfalls könnten sich gewissenhafte Menschen selbst in die Strafbarkeit hinein komplementieren.

\section{Vermeidbarkeit}

35 Das Bundesgericht setzt für die Fahrlässigkeitshaftung weiter voraus, dass der Erfolg vermeidbar war. "Dabei wird ein hypothetischer Kausalverlauf untersucht und geprüft, ob der Erfolg bei pflichtgemässem Verhalten des Täters ausgeblieben wäre. Für die Zurechnung des

$76 \overline{\text { Urteil des Bundesgerichts 6B_1091/2016 vom }}$ 18. Mai 2017 E. 3.2.3. 
Erfolgs genügt, wenn das Verhalten des Täters mindestens mit einem hohen Grad an Wahrscheinlichkeit die Ursache des Erfolgs bildete.»77 In der Sache geht es hier um das sorgfaltspflichtgemässe Alternativverhalten ${ }^{78}$ resp. den Pflichtwidrigkeitszusammenhang79, der teilweise als Problem der objektiven Zurechnung betrachtet wird. Es muss dem Täter somit eine Handlungsalternative aufgezeigt werden, welche den Erfolg abgewendet hätte. Dieser Prüfschritt ist trivial bei sinnlosen Tathandlungen, wie etwa dem wiederholten Überfahren eines zur Vernichtung in Brand gesetzten Müllhaufens mit einem Fahrzeug, welches nicht nur den Tank mit Benzin, sondern auch den Kofferraum mit Munition gefüllt hatte. ${ }^{80}$ Hier besteht die Vermeidealternative nicht darin, den eigenen Müll vorsichtiger zu verbrennen oder anschliessend sorgfältiger mit dem Fahrzeug darüberzufahren, sondern das Verhalten komplett zu unterlassen. Schwieriger zu beurteilen ist die Vermeidbarkeit bei risikoträchtigen, aber sozial erwünschten oder zumindest geduldeten Tätigkeiten wie dem Autofahren oder Entzünden von Feuerwerk. Hier ist dem Täter eine Vermeidealternative innerhalb des erlaubten Risikos (dazu sogleich unten 4.) aufzuzeigen. ${ }^{81}$

36 Im Herdplatten-Fall und im HeizungsFall kann das erfolgsvermeidende Verhalten somit nicht darin bestehen, auf die Benutzung der Herdplatten bzw. der

77 BGE 130 IV 7 E. 3.2.

78 Vgl. etwa Seelmann/Geth (Fn. 60), N 483.

79 Statt vieler Bernd Heinrich, Strafrecht Allgemeiner Teil, 3. Auflage, Stuttgart 2012, N 1042 ff.

80 Vgl. Man drives van carrying ammunition over Missouri field fire, Los Angeles Times vom 14. Oktober 2015.

81 Plastisch Rutz (Fn. 52), S. 258, 365 f.
Heizung ganz zu verzichten. Ebenso wenig kann verlangt werden, dass in der Küche bzw. im Keller überhaupt keine brennbaren Materialien gelagert werden. Vielmehr wäre den Tätern aufzuzeigen, welche Vorsichtsmassnahmen bei der Lagerung der fraglichen Gegenstände sowie der Anschaltung des Herds bzw. der Heizung zu ergreifen gewesen wären.

\section{Erlaubtes Risiko}

37 Viele sozial nützliche Tätigkeiten sind vorhersehbar mit Gefahren verbunden, die sich auch bei Einhaltung von Sicherheits- und Sorgfaltsstandards nicht ganz bannen lassen. Das gilt etwa für den Strassenverkehr, riskante Sportarten, den Betrieb von Küchenherden oder den Einsatz von Lithium-Akkus. ${ }^{82}$ Wollte man die Risiken ausschalten, müsste man die Tätigkeit verbieten. Gemäss Bundesgericht tritt deshalb beim erlaubten Risiko «an die Stelle des Verbots jeglicher Gefährdung das Gebot, die Gefahr auf dasjenige Minimum einzuschränken, das gar nicht oder nur mit unverhältnismässigem Aufwand ausgeschlossen werden kann, wenn man die entsprechende Tätigkeit überhaupt zulassen will».83

38 Im Umgang mit Brandgefahren kommt dem erlaubten Risiko eine grosse Bedeutung $\mathrm{zu}$, weil von vielen nützlichen (Smartphones, E-Bikes, Cheminée) oder sogar lebensnotwendigen Gegenständen (Heizung, Kochherd) Brandgefahren ausgehen. Dennoch wird dem erlaubten Risiko bei der fahrlässigen Verursachung einer Feuersbrunst im privaten Bereich oft zu wenig Beachtung geschenkt. Dies gilt insbesondere dann, wenn die Verlet-

82 Farag-Jaussi (Fn. 1), S. 144 f.

83 BGE 134 IV 193 E. 7.2. 
zung einer Sorgfaltspflicht mit der Verletzung einer ausserstrafrechtlichen Sorgfaltsnorm begründet wird. ${ }^{84}$ Sowohl bei den kantonalen Feuer- und Brandschutzbestimmungen als auch bei den Brandschutzvorschriften der VKF handelt es sich um präventive Vorschriften, welche ihr Ziel von maximalem Schutz nur auf Kosten der Praktikabilität erreichen können. Sie stellen in der Regel überhöhte, teilweise unrealistische Anforderungen an das Verhalten von Privatpersonen. Das Strafrecht demgegenüber muss einen Interessenausgleich inkorporieren. Nur die Verletzung elementarster Sorgfaltsregeln kann zugleich auch die Grundlage für ein strafrechtliches Unrecht darstellen. 85 Handlungen innerhalb des erlaubten Risikos können kein Unrecht darstellen. ${ }^{86}$

39 Als konkretes Beispiel kann die im Heizungs-Fall herangezogene Regelung dienen: Ziff. 7.3 Abs. 3 Brandschutzrichtlinie betreffend wärmetechnische Angaben bestimmte, dass leicht entzündbare Stoffe nicht im Heizraum gelagert werden dürfen. 87 Dass mit der Einhaltung dieser Vorschrift Brände verhindert werden können, liegt auf der Hand.

84 Donatsch (Fn. 50), S. 127; siehe auch Christof Riedo/Franz Riklin, Die strafrechtliche Verantwortung des Planers, in: Hubert Stöckli/Thomas Siegenthaler (Hrsg.), Planerverträge, Verträge mit Architekten und Ingenieuren, 2. Auflage, Zürich 2019, S. 941-966, N 21.78 f.

85 Vgl. Stratenwerth (Fn. 51), N 30 ff.; siehe auch BSK StGB I-Niggli/Mäder, Art. 12 N 86 m.W.H.

86 Urteil des Bundesgerichts 6S.728/1999 vom 6. März 2001 E. 3, wo das erlaubte Risiko als Grenze der objektiv geforderten Sorgfalt bezeichnet wird.

87 Vereinigung Kantonaler Feuerversicherungen (VKF), Brandschutzrichtlinie 25-03, Wärmetechnische Anlagen, vom 26. März 2003 (nicht mehr in Kraft); Entscheid des Kreisgerichts BernLaupen vom 10. Juni 2005, Sammelstelle Gerichtsentscheide (SG) 2006 Nr. 1589, Ziff. 21.
Allerdings stellt sich die Frage, wie ein Holzofen im Winter vernünftig betrieben werden soll, wenn kein brennbares Material in der Nähe gelagert werden darf. Das Beheizen der Familienwohnung als elementares und alltägliches Bedürfnis muss auf eine praktikable Art und Weise ausgeübt werden können. Eine derart praxisferne Verhaltensnorm kann nicht als Grundlage für einen strafrechtlichen Schuldvorwurf dienen. ${ }^{88}$

40 Im Herdplatten-Fall wurde der Beschuldigten vorgeworfen, eine Fritteuse sowie Textilien auf dem bzw. in der Nähe des Herdes gelagert zu haben. Das Abstellen einer Fritteuse auf oder unmittelbar neben einer Herdplatte liegt normalerweise im Bereich des erlaubten Risikos, muss für eine sinnvolle Nutzung doch möglich sein, diese direkt unter dem Dampfabzug zu platzieren. Auch das kurzfristige Lagern von Textilien wie Putzlappen oder Handtüchern in der Nähe der Herdplatten fällt unter das erlaubte Risiko, da der Kochherd in aller Regel in die Küchenkombination, welche u.a. als Ablage gebraucht wird, integriert ist.

41 Im Möbelpolitur-Fall musste sich das Bundesgericht nicht mit dem erlaubten Risiko beschäftigen, weil es bereits die Vorhersehbarkeit verneint hatte. Doch wären auch hier die Verwendung und unbeaufsichtigte Lagerung eines uneingeschränkt auf dem Mark zugelassenen Putzmittels im Rahmen des erlaubten Risikos gewesen. 89

$88 \overline{\text { Gleiches gilt etwa für Art. 3.2. Ziff. } 13 \text { letzter Satz }}$ der Brandschutzrichtlinie 12-15, welcher zum Umgang mit elektrischen Energieverbrauchern festhält: «Die Herstellerangaben sind einzuhalten.»

89 In concreto enthielt die spezielle Möbelpolitur brandgefährliches Leinöl und darf mittlerweile in der Schweiz so nicht mehr verkauft werden: Fritz 


\section{Fazit}

42 Die drei Fallbeispiele - der Möbelpolitur-, der Herdplatten- und der HeizungsFall - zeigen, dass Brandgefahren ein fester Bestandteil unseres Alltags sind. Kommt es im privaten Bereich zu einem ungewollten Brand mit Sach- oder Personenschaden, stellt sich im Einzelfall die Frage, ob die Schwelle für die fahrlässige Verursachung einer Feuersbrunst (Art. 222 StGB) überschritten ist, oder mit anderen Worten: Ob ein strafrechtlich relevantes Unrecht oder ein hinzunehmendes Unglück vorliegt.

Kern jedes Fahrlässigkeitsdelikts ist die Sorgfaltspflichtverletzung. Als Ausgangspunkt wird, wo solche vorhanden sind, auf besondere Sorgfaltsnormen abgestellt, was durchaus sinnvoll sein kann. Besondere Sorgfaltsnormen sind erfahrungsbasiert und auf die Verhinderung typischer, regelmässig auftretender Gefahren zugeschnitten. Werden für die Bestimmung der Sorgfaltspflicht von Privatpersonen im Umgang mit Feuergefahren kantonale Brand- und Feuerschutzgesetze oder die Brandschutzvorschriften der VKF berücksichtigt, vermag dies der Einzelfallgerechtigkeit aus drei Gründen jedoch oft nicht zu genügen: Erstens handelt sich dabei um ausserstrafrechtliche Vorschriften für den präventiven Brandschutz, welche Maximalanforderungen an das Verhalten von Privatpersonen stellen. Das strafrechtlich erlaubte Risiko stimmt oft nicht mit dem zulässigen Risiko von ausserstrafrechtlichen Normen überein und muss von den Gerichten deshalb stets im Einzelfall festgelegt werden. Nur die Verletzung elementars- ter Sorgfaltsregeln kann zugleich auch die Grundlage für ein strafrechtliches Unrecht darstellen. Zweitens muss eine Vorschrift gewisse inhaltliche Mindestanforderungen erfüllen, um als strafrechtliche Sorgfaltsnorm zu gelten. Die oft als reine Erfolgsverhinderungspflichten gefassten Brandschutzvorschriften der Kantone und der VKF erfüllen diese Mindestanforderung in der Regel nicht. Erfolgt eine Verurteilung alleine gestützt auf so eine Vorschrift, wird die Sorgfaltspflicht in unzulässiger Weise mit dem allgemeinen Gefahrensatz gleichgesetzt. Stattdessen ist es Aufgabe des Gerichts, der beschuldigten Person unter Berücksichtigung aller Umstände des Einzelfalls aufzeigen, welches Verhalten in concreto vorsichtig bzw. sorgfältig gewesen wäre. Drittens ist es auch fragwürdig, zur Begründung eines strafrechtlichen Fahrlässigkeitsvorwurfs auf Normen einer Vereinigung wie der VKF abzustellen, welche ein manifestes wirtschaftliches Interesse an der Verhinderung von Feuerschäden hat.

Thut, «Burestobe»-Brand: Schuld waren selbstentzündbare Putzlappen, in: aargauerzeitung.ch vom 13. März 2015. 